\title{
CONSTRUCTION OF AN ACCELERATED AGING TEST SYSTEM FOR SERIES CONNECTION BATTERY PACK
}

\author{
Hao Liu ${ }^{1} 凹\left(D\right.$, Jikai Bi ${ }^{2}$, Jae-Cheon Lee ${ }^{3}$
}

${ }^{1}$ Assistant Professor, Department of Mechanical Engineering, Keimyung University, Daegu (42601), South Korea.

${ }^{2}$ Ph. D Program, Graduate School of Mechanical Engineering, Keimyung University, Daegu (42601), South Korea.

${ }^{3}$ Professor, Department of Mechanical engineering, Keimyung University, Daegu (42601), South Korea

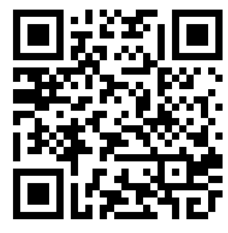

\section{ABSTRACT}

Nowadays, Lithium-ion batteries are widely used in various aspects, such as mobile electronic devices, mobility, EVs, and so on. Exactly to estimate State of Health (SoH) and Remaining Useful Life (RUL) becomes more and more necessary for realistic applications. Accelerated aging test can provide reliable experimental data for research of SoH estimation. An accelerated aging test system for a battery pack was designed in the research, which included hardware design and programming of test system control and monitoring. After establishment of the test system, several test cycles were implemented, and the acquired data indicated that the developed aging test system worked very well and can be used for degradation experiment of the Lithium-ion battery pack in future work.

Received 15 December 2021

Accepted 30 December 2021

Published 26 January 2022

\section{CorrespondingAuthor}

Hao Liu,

liuhao@kmu.ac.kr

DOI 10.29121/IJOEST.v6.i1.2022.272

Funding: This research received no specific grant from any funding agency in the public, commercial, or not-for-profit sectors.

Copyright: (C) 2022 The Author(s). This is an open access article distributed under the terms of the Creative Commons Attribution License, which permits unrestricted use, distribution, and reproduction in any medium, provided the original author and source are credited.

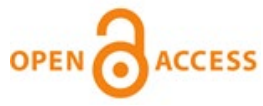

Keywords: Accelerated Aging Test, Lithium-Ion Battery Pack, Battery Internal Resistance. Test System Design, State of Health (SoH)

\section{INTRODUCTION}

With use of limited fossil fuel and climate change, demand of green and renewable energy sources is increasing greatly and continuously $\mathrm{Hu}$ et al. (2020). Secondary batteries, especially Lithium-ion batteries, have been widely used in various aspects, such as electronic devices Vetter et al. (2005), mobility, EVs Lin et al. (2015), Rezvanizanian et al. (2012). However, performance of Lithium-ion batteries degrades gradually with repetitive operation of charge and discharge Hu et al. (2020), Kai et al. (2008). Many researchers have focused on this field and developed estimation algorithms of battery aging, such as State of Health (SoH) and Remaining Useful Life (RUL) Xu et al. (2013), Gregory (2016). It is significantly important to use realistic battery aging data to validate those algorithms. There are some publicized data set Saha and Goebel (2007), CALCE of Lithium-ion battery in internet, one of which is provided by NASA Ames Prognostics Data Repository and has been used by many researchers. Most of these datasets are relative to single cell. However, we are going to investigate SoH prediction for a Lithium-ion battery pack. Thus, the aim of the research is to establish a set of accelerated aging test system for a battery pack that consists of two batter cells in series connection. The parameters of accelerated aging test can be set in the developed test system. During test period, voltage and current of battery cells in charge and discharge processes can be obtained and, meanwhile, internal resistance (IR) of the cells is acquired too. We can utilize these data 
sets to estimate SoH of the battery pack or to predict its RUL based on some algorithm.

The layout of the paper is as below. The test system designed includes hardware design and programming of control as well as monitoring, which are presented in the section 2 and 3, respectively. After establishing the test system, we did several cycle experiments and demonstrated a typical data set in the section 4 . Conclusions are given in the last section.

\section{DESIGN OF HARDWARE ARCHITECTURE}

Repeating charge and discharge cycles results in accelerated aging of the batteries, meanwhile, some parameters of batteries are measured to evaluate the batteries for the future and to ensure safety of entire experiment process. Figure 1 shows the architecture of accelerated aging test system for a battery pack, whose structure can be divided into three parts, a battery pack of two cells with serial connection, test and measurement instruments, and electrical circuit control part. The electrical circuit control part changes connection between the battery pack and the instruments. The battery pack, the object in the research, consists of two 18650 Lithium-ion batteries (cell 1 and cell 2) connected in serial. It will be described in detail in the following contents how to design hardware architecture. Table 1 gives typical specifications of the instruments shown in Figure 1.

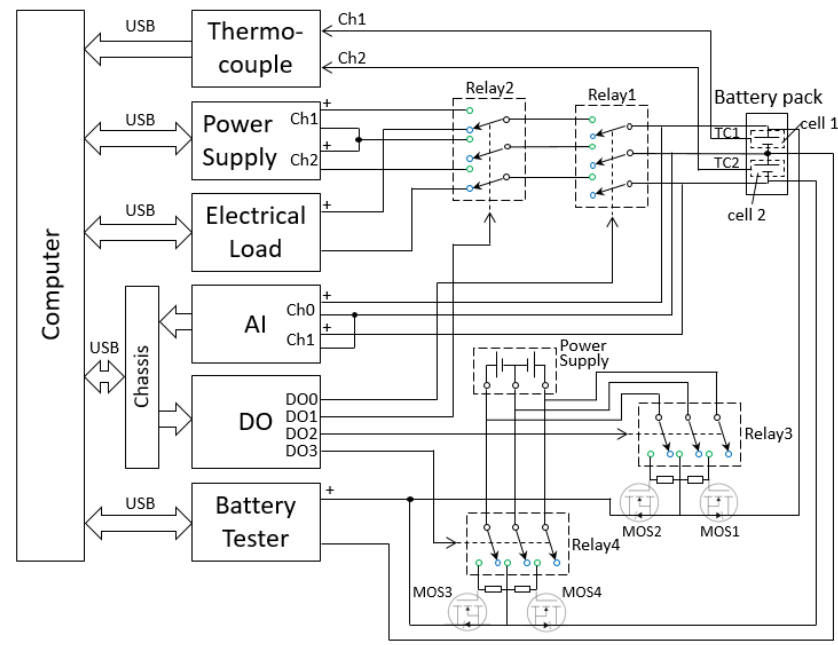

Figure 1 Architecture of accelerated aging battery test system for a battery pack

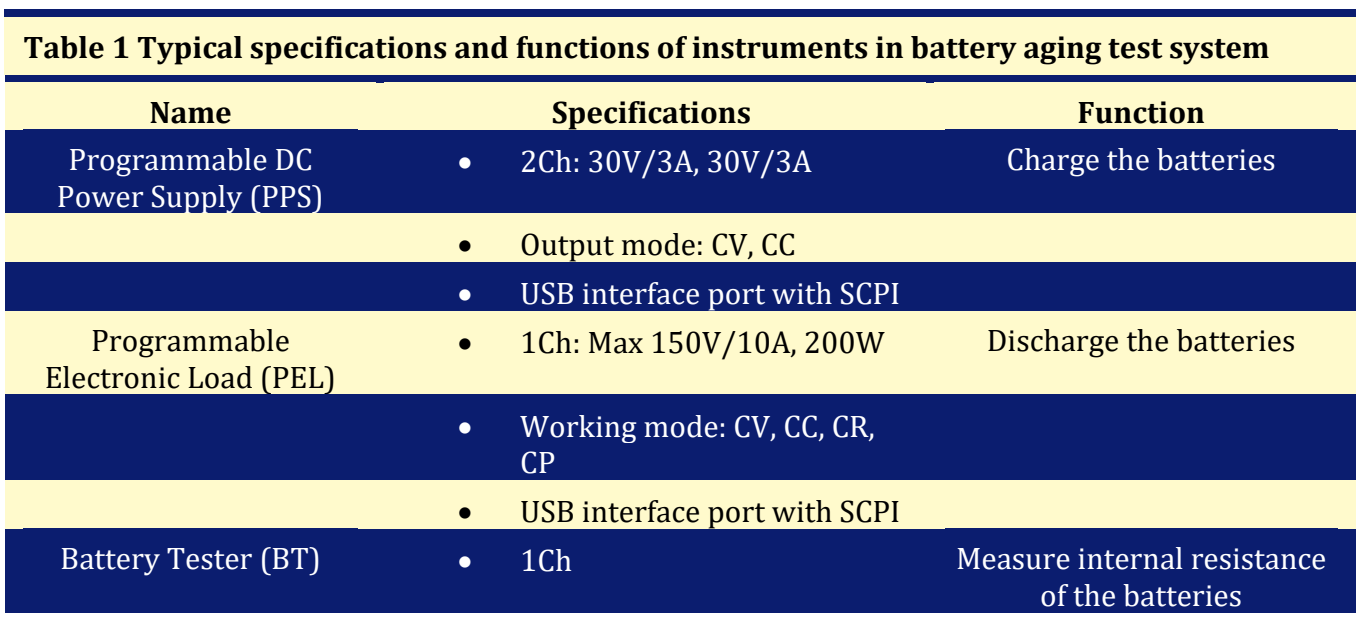


- Voltage: $0.01 \mathrm{mV} \sim 400.00 \mathrm{~V}$

- Resistance: $0.1 \mu \Omega \sim 3.2 \mathrm{k} \Omega$

- Trigger: internal or external

- USB interface port with SCPI

- $\quad 32 \mathrm{Ch}$ single-ended input

Measure voltages of the

Analog Input (AI)

16Ch differential input batteries

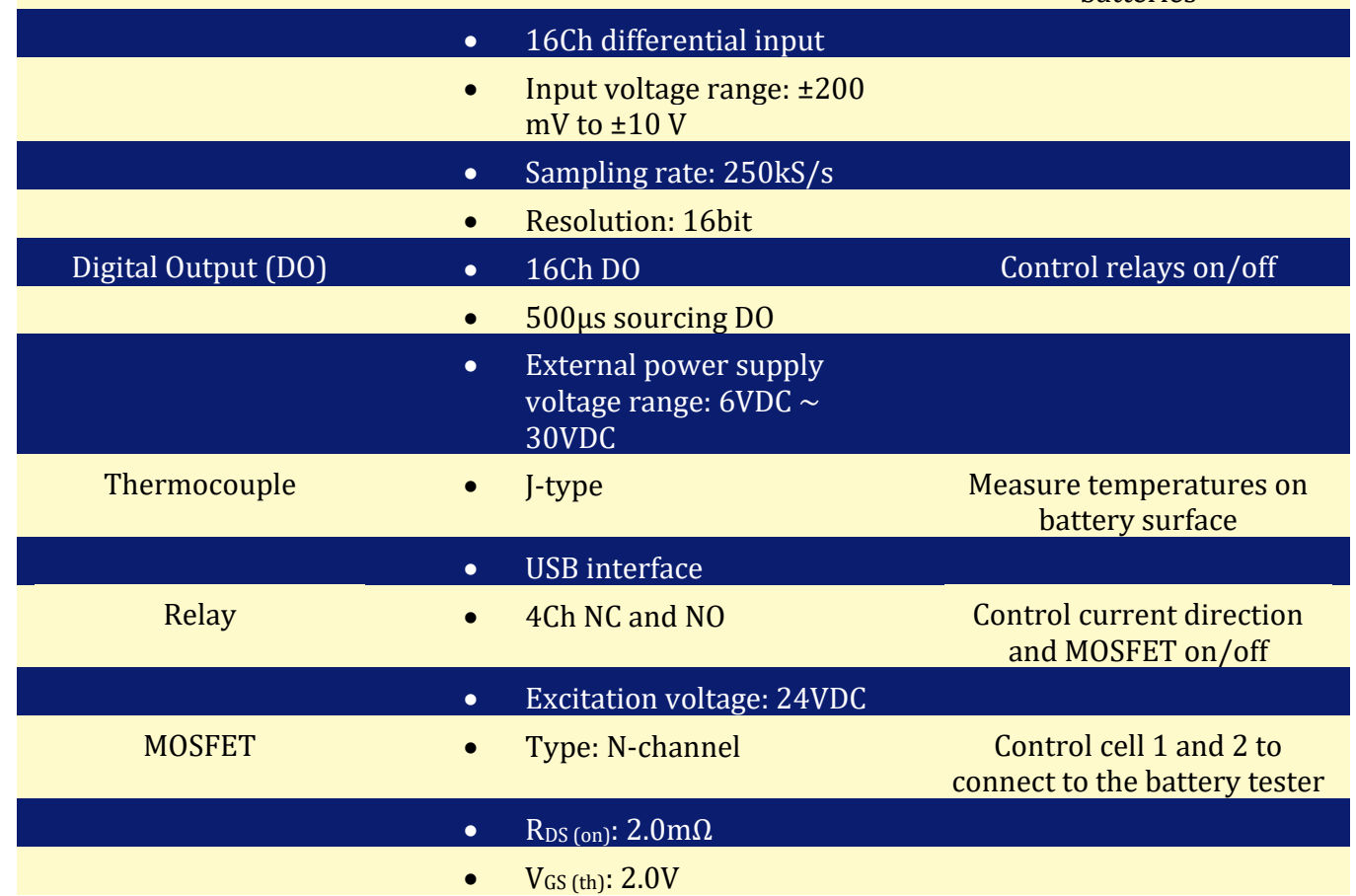

\subsection{REALIZATION OF CHARGE AND DISCHARGE FUNCTIONS}

A Programmable DC Power Supply (PPS) with two output channels was used to charge a battery pack, while a Programmable Electronic Load (PEL) was served as a load to discharge electricity from the battery pack. Relay 1 and relay 2 control whether the battery pack connects with PPS or PEL so as to implement charge or discharge process. In the normal state, in which the relays 1 and 2 are off, the battery pack does not connect with PPS or PEL. However, if switch on the relay 1 the battery pack is connected with the PEL, and discharge can be executed for two cells in serial connection by the same discharge current. The function of the relay 2 is to select charge or discharge connection with the battery pack. If the relay 2 is on the cells 1 and 2 are designed to electrically connect with channel 1 and 2 of the programmable power supply, respectively. Note that two channels of PPS are set as serial output mode, namely that the negative terminal of the channel 1 is shorted with the positive terminal of the channel 2 .

\subsection{REALIZATION OF MEASUREMENT OF INTERNAL RESISTANCE}

In order to evaluate degree of degradation of the battery pack, a battery tester (BT) for measurement of internal resistance (IR) for batteries was introduced in the test system. It was found that the IR of the Li-ion battery could not be accurately measured when the battery is in charge process. As a result, switches between the battery pack and the BT are necessary to ensure accurate data measurement and to change circuit in order to acquire individual IRs of two cells. However, there are two problems which should be solved when we design circuit connected to the battery 
tester. One problem is how to reduce contact resistance in the internal resistance measurement circuit, while other problem is how automatically to alter the connection circuit so that the BT with only one input channel could measure IRs of two batteries by turns without causing short of the batteries.

The first problem is solved by using MOSFET as switch because it has very low resistance $\left(\mathrm{R}_{\mathrm{DS} \text { (on) }}\right)$ between drain and source when the control voltage is greater than gate threshold voltage $\left(\mathrm{V}_{\mathrm{GS}}(\mathrm{th})\right)$. Another reason of using MOSFET is that $\mathrm{R}_{\mathrm{DS} \text { (on) }}$ is stable and not fluctuant, provided that the voltage at gate terminal is stable. In contrast to this, contact resistance between contacts of a relay is not stable with repetitive on/off operation. Moreover, the measurement branch points of the BT are not to locate on the path between relay 1 and 2, but directly to contact with three lead lines of the battery pack in order to reduce influence of resistance inside the circuit.

In order to solve the second problem, in the design as shown in Figure 1, the negative terminal of the BT is connected with the central lead wire of the battery pack, while the positive terminal of the BT is connected with the positive and negative terminals of the battery pack via four MOSFETs. If MOS1 and MOS2 in on state, MOS3 and MOS4 in off state, the positive terminal of the BT connects with the positive of the battery pack. Thus, the BT can measure IR of the cell 1. And vice versa, if MOS1 and MOS2 in off state, MOS3 and MOS4 in on state, the positive terminal of the BT connected with the negative of the battery pack, which indicate that IR of cell 2 can be measured. But the measured voltage of the cell 2 by the BT is displayed in negative value. The reason why using two MOSFETs in one wire as a switch is that there exists a body diode in a MOS, which can allow reverse current to flow if the battery voltage is greater than body diode forward voltage $\left(V_{S D}\right)$, yielding serious short accident. As a result, two MOSFETs are reversely connected in serial, which can prevent short. Note that four MOSFETs are never be switched on at the same time, otherwise short accident would happen. One independent power supply with double voltages is utilized to provide the gate voltages to either MOS1 and MOS2 or MOS3 and MOS4 via the relay 3 or the relay 4.

\subsection{FUNCTION REALIZATION OF CONTROL AND MONITORING}

In order to realize the functions of charge/discharge and IR measurement, four relays are used in the battery pack aging test system. The roles of the relays 1 and 2 are to determine whether the battery pack is isolated from the PPS or the PEL and whether it connects with the PPS or the PEL to execute charge or discharge process. Moreover, functions of the relays 3 and 4 are to isolate the battery pack from the BT and to establish independent connections between the BT and the cell 1 or the cell 2. A digital output (DO) device, whose four output channels are designated, controls the four relays. A 24VDC power should be provided to the DO device so that it can directly drive relays.

An analogue input (AI) device is utilized to measure voltages of the cell 1 and 2 . The measurement points are directly set at the battery pack in order to get rid of effect of voltage drop along lead wire or across contactors of relays. Since the AI device shares a common ground for all single-ended channels, the positive terminals of AI channel 1 and 2 are set to connect with the positive and negative of the battery pact, respectively. Thus, the AI channel 1 measures voltage of the cell 1 while the channel 2 acquires that of the cell 2 but with negative value due to inverse connection of the channel 2 on the cell 2 .

Li-ion batteries are sensitive to temperature and too high temperature easily causes heat out of control. Therefore, it is very significantly important to monitor 
temperature variation of two cells during charge and discharge processes. Two thermocouples with USB interface are attached on surfaces of two cells, respectively. Finally, the entire accelerated aging test system for the battery pack was set up as shown in Figure 2.

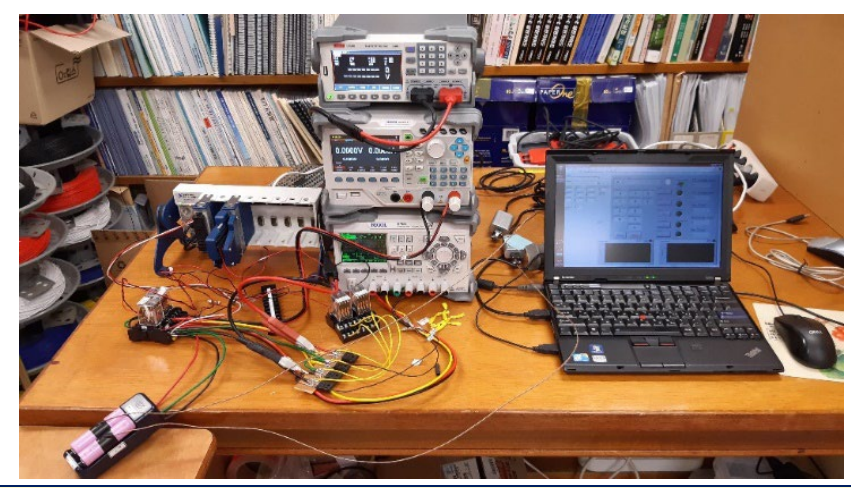

Figure 2 Photo of accelerated aging test system for the battery pack

\section{DESIGN OF CONTROL LOGIC}

According to the test procedure of battery data set [Saha and Goebel (2007), one battery test cycle comprised two stages, charge and discharge. Charge process was carried out in CC mode until the battery voltage reached a certain setting voltage $(4.2 \mathrm{~V})$ and then continued in $\mathrm{CV}$ mode until the charge current reduced to a certain current $(20 \mathrm{~mA})$. For the sake of safety, the rest process was added after charge stage as well as after discharge in our accelerated aging test. The control program was written based on the platform of LabVIEW, considering convenience of interface with hardware.

Figure 3 (a) illustrates the main flowchart of the control program, which is made of sequential, parallel, and loop structure. The loop structure is to execute cycle of charge and discharge processes. In each cycle, charge and discharge stages as well as rest stage are organized sequentially. Meanwhile, some important variables, such as time, cell voltages, charge/discharge current, cell internal resistance, should be simultaneously measured and recorded in data files. The cell voltages can be measured via AI device and current in charge and discharge stages can be read from the PPS and PEL, respectively. The BT can measure internal resistance of two cells only in the discharge and rest processes. At the same time, these measured data is written in an ASCII file.

As mentioned in 2.2 section, the relays 3 and 4 provide gate voltage to the four MOSFETs, so the BT can separately connect to only one cell and measure its IR. Any short connection of the cells must be strictly avoided. Therefore, the detail steps of IR measurement process shown in Figure 3(b) indicates that a waiting time is attached after each action of relay on/off and IR measurement to guarantee no short connection even in an instant. These steps repeat until discharge or rest processes finish.

Figure 3 (c) and Figure 3 (d) illustrate flowcharts of CC and CV charge stages. Considering difference of the two cells in the battery pack, it is designed that independent charge is executed for them, which is like BMS function. The setting parameters, such as voltage and current, should be sent to the PPS before the charge processes starts. During the charge process, the voltages across the cells and the charging currents are under continuous monitoring. If the voltage across a cell in the CC charge process reaches the setting voltage, its CC charge will stop and wait until another cell finishes CC charge. Similarly, in the CV charge process, if the charge 
current of a cell becomes less than the setting current, its CV charge will stop too and wait until another cell finishes this process.

On the other hand, the battery pack made by serial connection of two cells is designed to experience CC discharge by the same current. The terminative condition of the process is that the voltage of any cell is less than the setting value, as shown in Figure $3(\mathrm{e})$. In the rest process, after switching off all the relays, nothing is done but wait, and the terminative condition depends on whether battery temperature decreases enough or whether the designated waiting interval passes, as shown in Figure 3 (f)

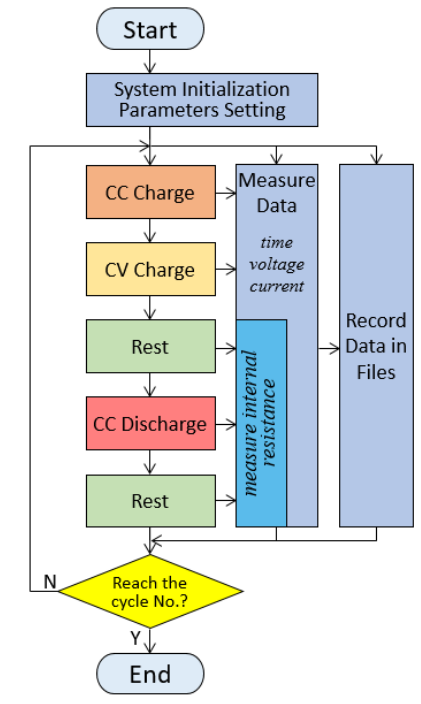

(a) Flowchart of main program

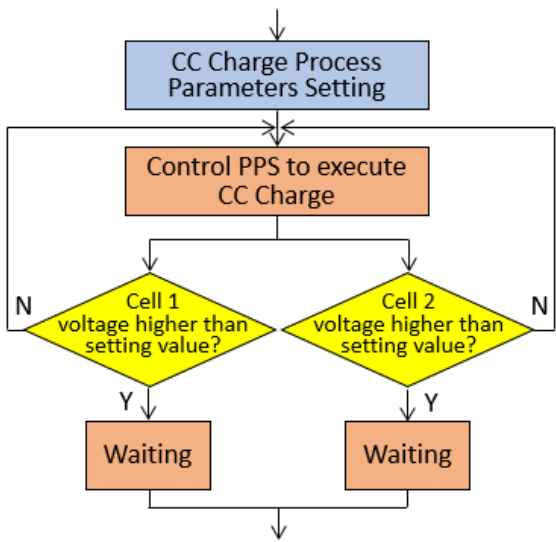

(c) Flowchart of CC charge

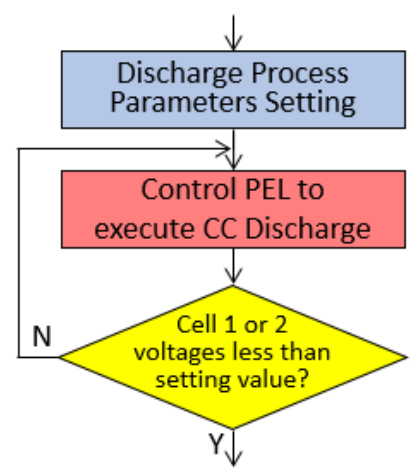

(e) Flowchart of CC discharge

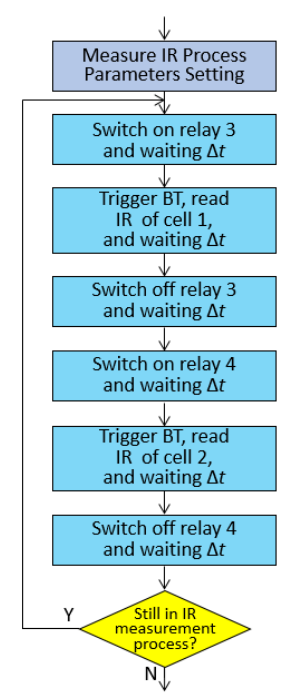

(b) Flowchart of cell IR measurement

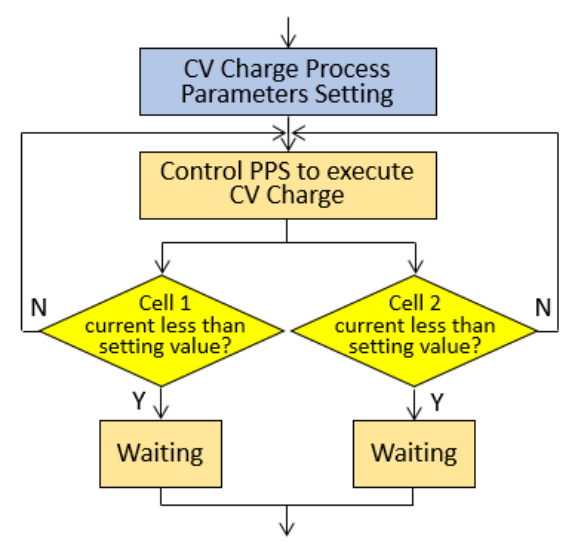

(d) Flowchart of CV charge

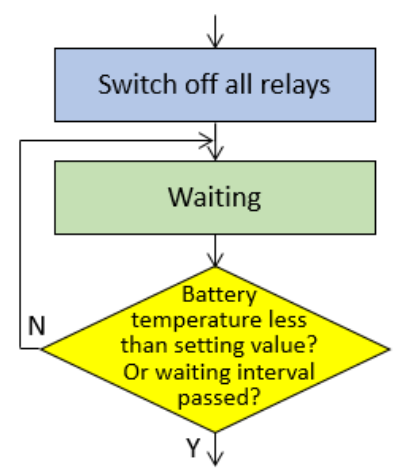

(f) Flowchart of rest 
According to hardware design of the test system mentioned in the section 2 and flowcharts of test program, operation states of the PPS, PEL, BT, AI device, TC device, DO device (relays) are summarized in Table 2. It can be seen that measurement of cell voltages and temperature is implemented throughout all stages. In other stages, the corresponding instruments work according to Table 2.

Table 2 Operation states of all components in each stage (Note: $\star$ : measure, 0: OFF, 1: ON)

\begin{tabular}{cccccccccccccccc}
\hline Stages & \multicolumn{2}{c}{ PPS } & PEL & \multicolumn{2}{c}{ AI } & \multicolumn{3}{c}{ TC } & \multicolumn{4}{c}{ DO } & \multicolumn{1}{c}{ BT } \\
& Ch1 & Ch2 & Ch0 & Ch0 & Ch1 & Ch0 & Ch1 & Ch3 & Ch & Ch & Ch & Ch0 \\
CC charge & ON & ON & OFF & $\star$ & $\star$ & $\star$ & $\star$ & OFF & OFF & ON & ON & OFF \\
\hline CV charge & ON & ON & OFF & $\star$ & $\star$ & $\star$ & $\star$ & OFF & OFF & ON & ON & OFF \\
\hline CC discharge & OFF & OFF & ON & $\star$ & $\star$ & $\star$ & $\star$ & OFF & OFF & OFF & ON & ON \\
\hline Rest & OFF & OFF & OFF & $\star$ & $\star$ & $\star$ & $\star$ & OFF & OFF & OFF & OFF & ON \\
\hline $\begin{array}{c}\text { Measure cell 1 } \\
\text { IR }\end{array}$ & OFF & OFF & OFF & $\star$ & $\star$ & $\star$ & $\star$ & OFF & ON & OFF & OFF & ON \\
$\begin{array}{c}\text { Measure cell 2 } \\
\text { IR }\end{array}$ & OFF & OFF & OFF & $\star$ & $\star$ & $\star$ & $\star$ & ON & OFF & OFF & OFF & ON \\
\hline
\end{tabular}

\section{REPRESENTATION OF TYPICAL ACQUIRED DATA}

The accelerated aging test system for the battery pack was established according to the section 2 and 3 . However, before a formal aging test is implemented, we should execute serval cycle for the battery pack in order to activate electrochemical performance of the battery cell. The cell was Lithium-ion 18650 battery with $2600 \mathrm{mAh}$. Several cycles test has been done. One typical cycle was selected, and its acquired data set was plotted and shown in Figure 4. The cycle sequentially included four stages: CC charge, CV charge, CC discharge, and rest. There was not rest stage after charging due to temperature of the battery pack was not high. It can be seen that it took about 2 hours to charge the battery pack and about one hour to discharge. The charge current represents in positive value while discharge current in negative on the figure. Cell temperature went up during CC charge since the current is $1.95 \mathrm{~A}(0.75 \mathrm{C})$ and then reduced in CV charge. However, it rapidly rose up in the beginning of CC discharge. Moreover, temperature sharply increased again in the end of this stage, and, at the same time, the cell voltages decreased abruptly as it lower than $3.2 \mathrm{~V}$. If investigating internal resistance, we can find that it gradually increases with increasing of depth of discharge. In addition, there exists offset value between the internal resistance of the cell 1 and that of the cell 2 since the two cells have different IR in initial state. Of course, more analysis can be implemented once more aging cycle test is done. In conclusion, data acquired by the accelerated aging test system is valid. 

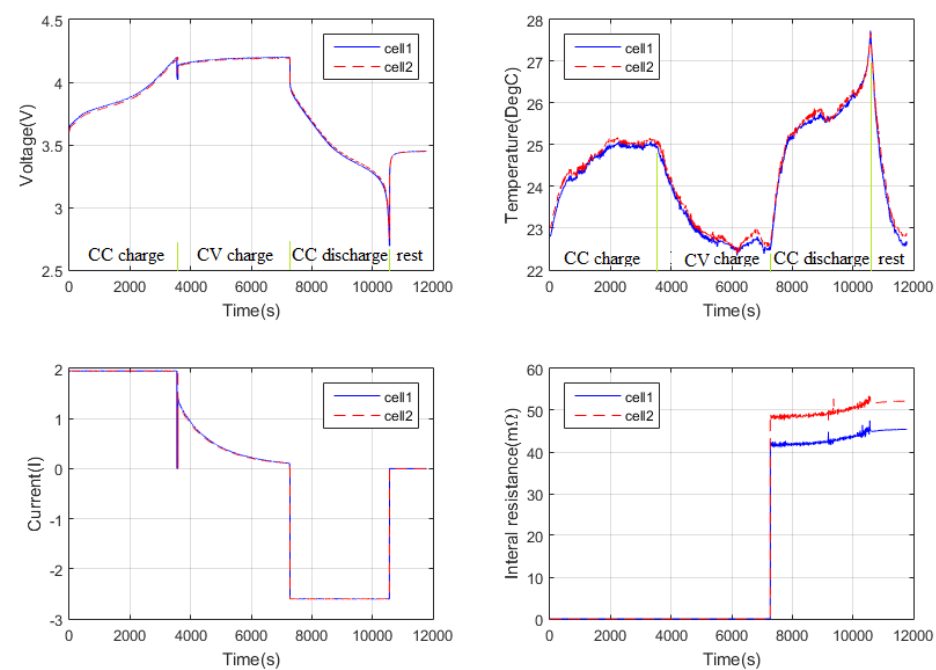

Figure 4 Typical test results: voltage, current, temperature, and internal resistance

\section{CONCLUSIONS}

The research proposed a design of accelerated aging test system for the battery pack, which consists of two cells in serial connection. This test system mainly comprises a programmable power supply, a programmable electronic load, and a battery internal resistance tester, and it can implement degradation cycle test of charge and discharge as well as measurement of internal resistance of two cells. Voltage, current, and temperature of two cells can be monitored individually too. A trail run of several cycles has been done by using the design test system and it is found that the measured data set is acceptable and valid. Next work is to utilize the developed test system to implement aging cycle test so that we can analyze obtained data to estimate SoH of the Lithium-ion battery pack.

\section{ACKNOWLEDGEMENTS}

Outcomes of this research are results of a study on the "Convergence and Open Sharing System" Project, supported by the Ministry of Education and National Research Foundation of Korea.

\section{REFERENCES}

Chen, Y., \& Huang, M. (2016). A Method of Battery State of Health Prediction based on AR-Particle Filter. SAE Technical Paper 2016-01-1212. Retrieved from https://doi.org/10.4271/2016-01-1212

Gregory, L. P. (2015). Battery Management Systems Volume I Battery Modeling. Artech House.

Gregory, L. P. (2016). Battery Management Systems Volume II Equivalent-Circuit Methods. Artech House

Hu, X. S., Xu, L., Lin, X. K., \& Pecht, M. (2020). Battery Lifetime Prognostics. Joule, 4(2), 310-346. Retrieved from https://doi.org/10.1016/j.joule.2019.11.018

Jiang, J. C., Zhang, C. P. (2015). Fundamentals and Applications of Lithium-ion Batteries in Electric Drive Vehicles. John Wiley \& Sons. Retrieved from https://doi.org/10.1002/9781118414798 
Kai, G., Bhaskar, S., Abhinav, S., et al. (2008). Prognostics in Battery Health Management. IEEE Instrumentation \& Measurement Magazine, 11(4), 3340. Retrieved from https://doi.org/10.1109/MIM.2008.4579269

Lin, C., Tang, A. H., \& Wang, W. W. (2015). A review of SOH estimation methods in Lithium-ion batteries for electric vehicle applications. Energy Procedia, 75, 1920-1925. Retrieved from https://doi.org/10.1016/j.egypro.2015.07.199

Rezvanizanian, S. M., Huang, Y. X., Chuan, J., \& Lee, J. (2012). A Mobility Performance Assessment on Plug-in EV Battery. International Journal of Prognostics and Health Management, 3(2), Retrieved from https://doi.org/10.36001/ijphm.2012.v3i2.1363

Saha, B., \& Goebel, K. (2007). Battery Data Set, NASA Ames Prognostics Data Repository, NASA Ames Research Center, Moffett Field, CA. Retrieved from https://ti.arc.nasa.gov/tech/dash/groups/pcoe/prognostic-datarepository/

Verena, K., Mårten, B., Göran, L. (2014). A support vector machine-based state-ofhealth estimation method for lithium-ion batteries under electric vehicle operation. Journal of Power Sources, 270(15), 262-272. Retrieved from https://doi.org/10.1016/j.jpowsour.2014.07.116

Vetter, J., Novak, P., Wagner, M. R., et al. (2005). Ageing mechanisms in lithium-ion batteries. Journal of Power Sources, 147(1-2), 269-281. Retrieved from https://doi.org/10.1016/j.jpowsour.2005.01.006

Vasan, A. S. S., Mahadeo, D. M., Doraiswam, R., Huang, Y., and Pecht, M. (2013) Pointof-care biosensor system. Center for Advanced LifeCycle Engineering (CALCE). https://calce.umd.edu/battery-data. 39-71 january 1

Xu, J., Mi, C. C., Cao, B. G., Cao, J. Y. (2013). A new method to estimate the state of charge of lithium-ion batteries based on the battery impedance model. Journal of Power Sources, 233(1), 277-284. Retrieved from https://doi.org/10.1016/j.jpowsour.2013.01.094 